\title{
Rationale and conditions for the requirement of chiral bioanalytical methods in bioequivalence studies
}

\author{
Juan José Torrado • María Blanco • Magí Farré • \\ Pere Roset • Alfredo García-Arieta
}

Received: 8 October 2009 / Accepted: 20 January 2010/Published online: 27 February 2010

(C) Springer-Verlag 2010

\begin{abstract}
Purpose The aim of the present work was to assess the need for chiral bioanalytical methods in bioequivalence studies.

Methods The samples from a bioequivalence study of two ibuprofen $2 \%$ oral suspensions that had shown bioequivalence for AUC and $\mathrm{C}_{\max }$, but not for $\mathrm{t}_{\max }$ (medians of 2.0 and
\end{abstract}

This manuscript represents the personal opinion of the author and does not necessarily represent the views or policy of the Spanish Agency for Medicines and Health Care Products.

\section{J. J. Torrado $\cdot$ M. Blanco}

Departamento Farmacia y Tecnología Farmacéutica,

Facultad de Farmacia, Universidad Complutense de Madrid,

Plaza Ramón y Cajal,

28040 Madrid, Spain

\section{Farré}

Unitat de Farmacologia Humana i Neurociències,

IMIM-Hospital del Mar,

Carrer Doctor Aiguader, 88,

08003 Barcelona, Spain

\section{Farré}

Universitat Autònoma de Barcelona,

Bellaterra (Cerdanyola del Valles),

08193 Barcelona, Spain

\section{P. Roset}

Laboratorios Gelos, Grupo Ferrer,

Carrer Joan XXIII, 10,

08950 Esplugues de Llobregat (Barcelona), Spain

\section{A. García-Arieta $(\bowtie)$}

Division of Pharmacology and Clinical Evaluation, General Subdirectorate for Human Use Medicines,

Spanish Agency for Medicines and Health Care Products,

C/Campezo 1, Edificio 8, Planta 2 A,

28022 Madrid, Spain

e-mail: agarciaa@aemps.es
$0.75 \mathrm{~h}$ ) with a non-chiral method were assayed with a chiral method to investigate whether there was an actual difference in the rate of absorption within the limits of $\mathrm{C}_{\max }$ and $\mathrm{AUC}$ bioequivalence.

Results The non-chiral method and the sum of concentrations of both enantiomers obtained with the chiral method gave a similar outcome $\left(90 \% \mathrm{CI} \mathrm{C}_{\max }\right.$ non-chiral: 82.77-96.09, sum of enantiomers: 82.19-98.23; 90\% CI $\mathrm{AUC}_{\mathrm{t}}$ non-chiral: 107.23-115.49, sum of enantiomers: 105.73-121.35). However, the chiral method showed differences in $\mathrm{AUC}$ and $\mathrm{C}_{\max }$ that resulted in non-bioequivalence for the individual enantiomers $\left(90 \% \mathrm{CI} \mathrm{C}_{\max } S\right.$-ibuprofen: 76.05-91.36, $R$-ibuprofen: 87.84-113.05; 90\% CI AUC $\mathrm{t}_{\mathrm{t}} S$ ibuprofen: 96.67-105.86, $R$-ibuprofen: 118.86-142.24). The differences in the pharmacokinetics of each enantiomer, and thus in the enantiomer concentration ratio, were dependent on the rate of absorption.

Conclusions Due to the fact that in bioequivalence studies the rate of absorption of the new product is unknown, chiral bioanalytical methods should be employed for chiral drugs, such as ibuprofen, whose enantiomers exhibit different pharmacodynamic characteristics and whose enantiomer concentration ratio might be modified by the rate of absorption, irrespective of whether the eutomer is the minor enantiomer or the similarity of the pharmacokinetics of the enantiomers at a given rate of absorption.

Keywords Ibuprofen - Bioequivalence - Enantiomers · Chirality $\cdot$ Regulatory requirements

\section{Introduction}

The need for chiral bioanalytical methods in bioequivalence studies is a controversial issue that has not been agreed upon 
by different regulatory agencies $[1,2]$. The FDA Guidance for Industry [2] recommends the measurement of the racemate using a non-chiral assay for bioequivalence studies. Measurement of individual enantiomers in bioequivalence studies is only recommended when all of the following conditions are met: (1) the enantiomers exhibit different pharmacodynamic characteristics, (2) the enantiomers exhibit different pharmacokinetic characteristics, (3) primary efficacy/safety resides with the minor enantiomer, and (4) non-linear absorption is present (as expressed by a change in the enantiomer concentration ratio with change in the input rate of the drug) for at least one of the enantiomers. In such cases, bioequivalence criteria should be applied to the enantiomers separately.

It is obvious that if enantiomers do not exhibit different pharmacodynamic characteristics, chiral bioanalytical methods are unnecessary. However, the other three conditions are debatable or difficult to understand.

It has been shown previously that even when the active (eutomer) and inactive enantiomer (distomer) exposure ratio is near unity, the non-chiral bioanalytical method may be biasing the results [3]. Obviously, if the eutomer were the minor enantiomer, the bias would be greater, but it does not mean that in other cases the bias is nonexistent.

The concept of non-linear absorption (expressed as a change in the enantiomer concentration ratio with a change in the input rate of the drug) has been confused frequently with non-linear pharmacokinetics (e.g. as required in the CPMP Notes for Guidance [1, 4]). Boni et al. [5] illustrated with etodolac that this non-linear absorption may occur in drugs with linear kinetics both for the racemic and the individual enantiomers.

In addition, it must be taken into account that for those drugs where a change in the rate of absorption produces a change in the enantiomer concentration ratio, it could be understood that these enantiomers exhibit different pharmacokinetic characteristics, at least at some rates of absorption.

The need for chiral bioanalytical methods in bioequivalence studies has been discussed from a theoretical point of view [6], or experimentally, changing the input rate artificially [5]. However, actual examples would be convenient to confirm if chiral bioanalytical methods are necessary in bioequivalence studies.

Ibuprofen is a chiral non-steroidal anti-inflammatory drug with the $S$-enantiomer possessing most of the beneficial anti-inflammatory activity [7]. Although enantiomer exposure ratio is near unity, the drug exhibits a complex and marked stereoselectivity in its pharmacokinetics [8]. In addition, according to the Biopharmaceutics Classification System (BCS) [9, 10], ibuprofen is a highly soluble at neutral $\mathrm{pH}$ class II drug with complete oral absorption, but whose rate of absorption is highly dependent on formulation and manufacturing factors $[11,12]$ or even on the interaction with antacids [13]. In other words, due to its low solubility at acidic $\mathrm{pH}$, different formulations may show different in vivo dissolution rates, which lead to different rates of absorption [14]. For these reasons it was chosen as a model drug in a previous [3] and the present work.

The aim of the present work was to challenge the validity of the present regulatory requirements on chiral bioanalytical methods in bioequivalence studies. For this purpose, the samples from a bioequivalence study of two ibuprofen $2 \%$ oral suspensions that had shown bioequivalence for AUC and $\mathrm{C}_{\max }$, but not for $\mathrm{t}_{\max }$, with a non-chiral method, were assayed with a chiral bioanalytical method to investigate an actual difference in rate of absorption within the limits of $\mathrm{C}_{\max }$ and $\mathrm{AUC}$ bioequivalence.

\section{Materials and methods}

Study design

A randomized, open-label, single dose, balanced, two-way crossover study was conducted with the approval of the Ethical Committee (CEIC-IMAS) and the Spanish Agency for Medicines and Health Care Products. Written informed consent was obtained from subjects after explaining the nature and purpose of the study. The 18 volunteers (nine males and nine females) were non-smokers; were determined to be in good health by physical examination, complete blood count, urinalysis, and serum test on hepatic and renal function; and refrained from taking any drug including OTC products for at least 1 week prior to or during the study. After fasting overnight for $8 \mathrm{~h}$, a dose of $600 \mathrm{mg}$ of ibuprofen $2 \%$ suspension was orally administered with $220 \mathrm{~mL}$ of water, either as a new test product (Laboratorios Gelos, Spain; batch: R-002, content: 102\%), or as the reference product (Dalsy, Laboratorios Knoll, Spain; batch: R-50, content: 103\%). Blood was drawn before dosing and at $0.25,0.5,0.75,1$, $1.5,2,2.5,3,4,6,8,10,12$ and $24 \mathrm{~h}$ after dosing through an in-dwelling catheter placed in an antecubital vein in the forearm. The blood samples were centrifuged, and the plasma was collected and stored at $-20^{\circ} \mathrm{C}$ until assayed. Seven days after this first administration, the alternative formulation was given to complete the sequence.

\section{Assay procedure}

Ibuprofen plasma concentrations were determined by the sponsor following a validated non-chiral method already described [15]. Subsequently, samples were assayed for ibuprofen enantiomers using a chiral AGP- $\alpha 1$ column of $100 \times 4 \mathrm{~mm}$ ID (Chrom Tech, Sweden) according to the method previously published [3]. Ibuprofen enantiomers were extracted from $0.6 \mathrm{~mL}$ of plasma (to which $0.15 \mathrm{~mL}$ 
of $3 \mathrm{~N} \mathrm{HCl}$ was added) with $5 \mathrm{~mL}$ of cyclohexane. After 15 min of vortexing, the samples were centrifuged at $1,200 \mathrm{~g}$ for $5 \mathrm{~min}$, and $3 \mathrm{~mL}$ of the organic phase was separated. The organic extracts were evaporated to dryness in a Savant Speedvac concentrator (Savant Instrument, USA) at $45^{\circ} \mathrm{C}$ under vacuum. The residue was then reconstituted with $1.5 \mathrm{~mL}$ of mobile phase and an aliquot of $100 \mu \mathrm{L}$ was injected into the HPLC.

The assay was performed with a modular Jasco (Japan) liquid chromatograph equipped with a DG-2080-53 3-line degasser, a LG 2080-02 ternary gradient unit, a PU-1580 pump, an AS2050 Plus autosampler (100 $\mu$ l sampling loop) and a UV-1575 UV-visible detector. Integration of the peaks was performed with the program Borwin 1.5 for PC (JMBS Developments). Separation and determination of the proportions of $R$ - and $S$-ibuprofen enantiomers were achieved with a chiral AGP column and UV detection at $220 \mathrm{~nm}$. The mobile phase was $990 \mathrm{~mL}$ phosphate buffer with orthophosphoric acid (pH 6.9)/10 mL acetonitrile/204 $\mu \mathrm{L} \mathrm{N}$, $\mathrm{N}$-dimethyloctylamine at a flow rate of $1.5 \mathrm{~mL} / \mathrm{min}$.

Under these conditions $R$ - and $S$-ibuprofen enantiomers had retention times of 2.2 and $3.5 \mathrm{~min}$ respectively. Resolution determined by the USP 29 method was of 2.1 . Figure 1 shows the chromatograms of standards of racemic ibuprofen (Fig. 1a), pure $S$-ibuprofen (Fig. 1b) and a representative test sample (Fig. 1c). Linearity of the method was studied in the range between 0.25 and $30 \mu \mathrm{g} / \mathrm{mL}$ of $R$ and $S$-ibuprofen $(0.25,0.5,1,5,10,15,20$ and $30 \mu \mathrm{g} / \mathrm{mL})$ using as reference compounds racemic ibuprofen and pure $S$-ibuprofen supplied by Shasun (India). The results were analysed by linear regression, and correlation coefficients obtained were always at least 0.98 for both enantiomers. The lower quantification limit was $0.25 \mu \mathrm{g} / \mathrm{mL}$ for both enantiomers with relative standard deviation of 7.4 and $7.0 \%$ for the $R$ - and $S$-ibuprofen enantiomers respectively. Accuracy (tested at 1,15 and $30 \mu \mathrm{g} / \mathrm{mL}$ ) ranged from 96.2 to $102.4 \%$. Inter-day precision during in-study validation (tested at $0.75,7.5$ and $22.5 \mu \mathrm{g} / \mathrm{mL}$ ) was not higher than
4.5 and $5.6 \%$ for the $R$ - and $S$-enantiomers respectively. Ibuprofen concentration data reported as racemic were obtained as the sum of the two enantiomers.

\section{Pharmacokinetics}

The estimation of the pharmacokinetic parameters was performed from the plasma concentration-time data by non-compartmental analysis using WinNonlin Professional, version 2.0 (Pharsight, USA). $\mathrm{C}_{\max }$ and $\mathrm{t}_{\max }$ were obtained directly from the plasma concentration-time data. The AUC to the last time with measurable concentration $\left(C_{t}\right)$ of the analyte $\left(\mathrm{AUC}_{0-\mathrm{t}}\right)$ was estimated by using the linear trapezoidal rule. The apparent elimination rate constant $\left(\mathrm{k}_{\mathrm{e}}\right)$ was calculated by least squares log-linear regression from data of the last sample time points of each plasma concentration-time curve. The AUC extrapolated to infinity $\left(\mathrm{AUC}_{0-\infty}\right)$ was determined by adding the extrapolated area $\left(\mathrm{C}_{\mathrm{t}} / \mathrm{K}_{\mathrm{e}}\right)$ to the $\mathrm{AUC}_{0-\mathrm{t}}$.

\section{Statistical analysis}

The two-way analysis of variance (ANOVA) for crossover design was used to assess the effect of formulations, periods, sequences and subjects within sequence on logarithmically transformed data of $\mathrm{AUC}_{0-\mathrm{t}}, \mathrm{AUC}_{0-\infty}$ and $\mathrm{C}_{\max }$ [16]. The parametric analyses of the data were performed with WinNonlin Professional. The comparison of enantiomers within each formulation was carried out with a similar methodology, considering $S$-enantiomer as test and $R$-enantiomer as reference.

\section{Results}

Figures 2 and 3 show the plasma concentration-time profile of total ibuprofen (sum of $S$ - and $R$-enantiomers) and that of the $S$ - and $R$-enantiomers of both formulations respectively. a

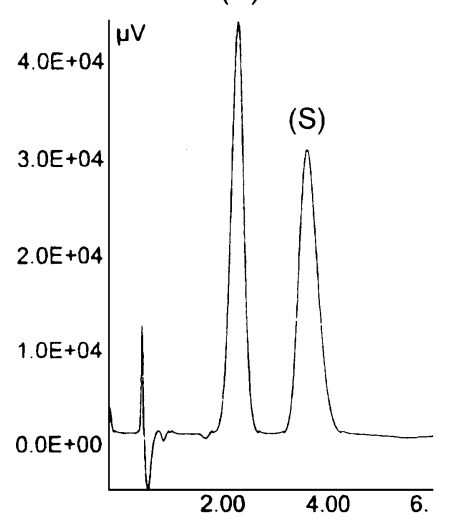

b

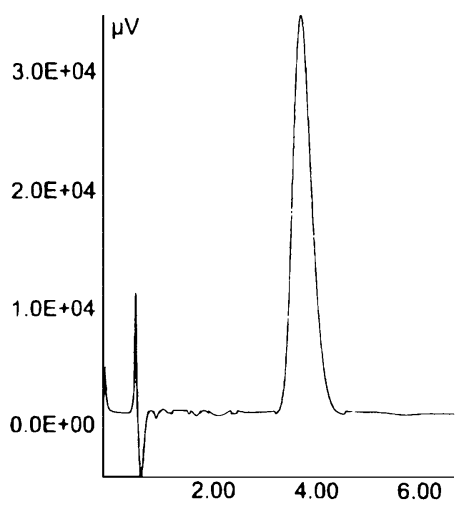

C

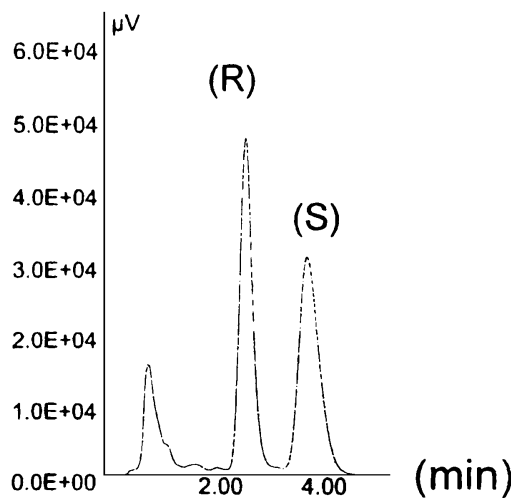

Fig. 1 HPLC chromatograms of standard racemic ibuprofen (a), $S$-ibuprofen (b) and a representative test sample (c) 


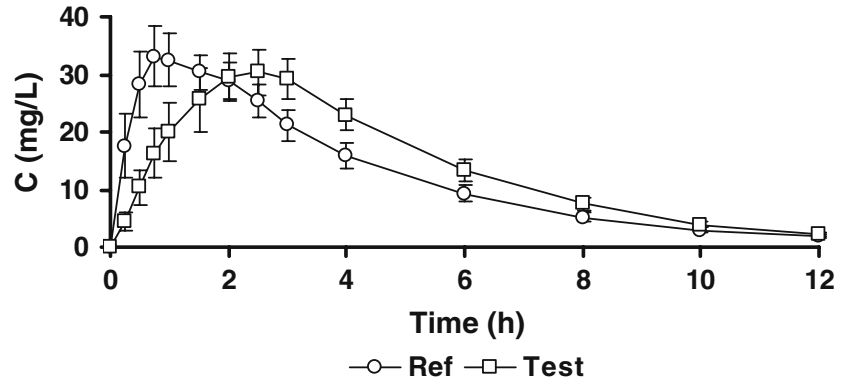

Fig. 2 Mean plasma concentration-time curve $(n=18)$ of racemic ibuprofen after oral administration of $600 \mathrm{mg}$ of ibuprofen in the reference and test formulations. Vertical bars show standard error of the mean

The bioequivalence analyses based on the original non-chiral bioanalytical method, the sum of the enantiomers and the individual $S$ - and $R$-enantiomers are summarised in Table 1 .

The test product showed bioequivalence to the reference product for $\mathrm{C}_{\max }$ and $\mathrm{AUC}$ when considering either the non-chiral concentrations or the sum of enantiomers. However, the $\mathrm{C}_{\max }$ of the eutomer ( $S$-ibuprofen) and the AUC of the distomer ( $R$-ibuprofen) were not bioequivalent.

The mean relative bioavailability of the sum of enantiomers of the test product was $13 \%$ higher for AUC and $10 \%$ lower for $\mathrm{C}_{\max }$ compared to the reference one, and these differences were statistically significant since their $90 \%$ confidence intervals did not include $100 \%$. The mean relative bioavailability of the eutomer of the test product was $1 \%$ higher for AUC and $17 \%$ lower for $C_{\max }$ than the reference one, and the difference was significant for $\mathrm{C}_{\max }$. Conversely, the mean relative bioavailability of the distomer of the test product was $30 \%$ higher for AUC and $0.4 \%$ lower for $\mathrm{C}_{\max }$, and the difference in AUC was significant. Obviously, the point estimates of AUC and $\mathrm{C}_{\max }$ of the racemate are located in between those of the enantiomers.

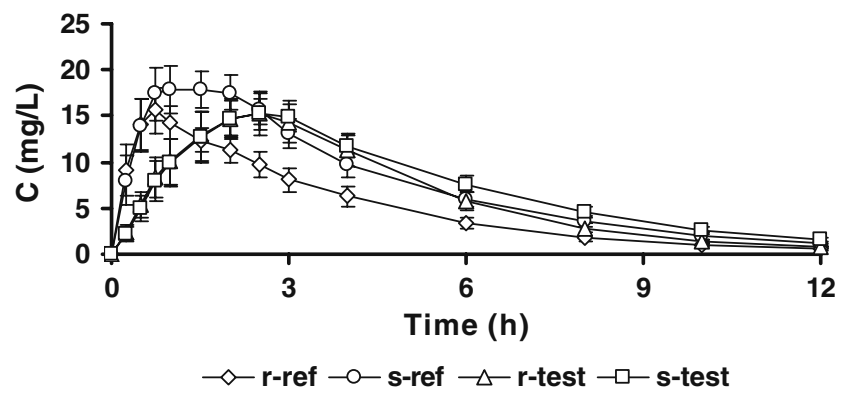

Fig. 3 Mean plasma concentration-time curve $(n=18)$ of ibuprofen enantiomers after oral administration of $600 \mathrm{mg}$ of ibuprofen in the reference and test formulations. Vertical bars show standard error of the mean
The ratio of $S$-enantiomer to $R$-enantiomer in each product for the main pharmacokinetic parameters, presented in Table 2, shows that $S$-ibuprofen is the overall prevalent enantiomer in plasma and those ratios were higher with the reference product. Moreover, the $S / R$ enantiomer ratio changed over time and showed a different profile between products, with higher eutomer/distomer ratio in the formulation with the faster rate of absorption (Fig. 4).

\section{Discussion and conclusions}

The test product showed a slower rate of absorption, with more than 30 min delay in $t_{\max }$, despite a similar $\mathrm{C}_{\max }$, and its marketing authorisation was rejected since, for ibuprofen, onset of action is considered clinically important. However, this criterion is somewhat subjective, and it might have been approved since $\mathrm{AUC}$ and $\mathrm{C}_{\max }$ were bioequivalent, and alternative metrics such as partial AUC [17] are not used due to their high variability. Furthermore, if this drug had been one for which onset of action was not clinically relevant, the test product would have been approved based solely on AUC and $\mathrm{C}_{\max }$. Figure 3 and Table 1, however, show that the difference in $\mathrm{C}_{\max }$ of the eutomer is larger and its $90 \%$ confidence interval lies outside the bioequivalence limits, which supports the rejection of the application. Importantly, this illustrates that even when $\mathrm{C}_{\max }$ is equivalent there is room for large differences in rate of absorption because $\mathrm{C}_{\max }$ is not a sensitive metric for detecting differences in rate of absorption $[18,19]$. These differences in rate of absorption may produce very different enantiomer ratios in products containing drugs with non-linear absorption as defined by the FDA [2].

Interestingly, the AUCs of the eutomer ( $S$-ibuprofen) were more similar between products, but the AUCs of the distomer ( $R$-ibuprofen) were more different when compared with the original non-chiral analysis or the sum of enantiomers. This could be considered as clinically irrelevant but shows a different biopharmaceutical quality.

The logical observation that the point estimates of the sum of enantiomers is between the point estimates of the enantiomers demonstrates again [3] that the results of chiral assay may be biased even when the eutomer is not the minor one. The difference in eutomer exposure is detected with less accuracy by non-chiral assay. This $6.5 \%$ shift in the $\mathrm{C}_{\max }$ point estimate changed the conclusion of the study despite the fact that the eutomer is the enantiomer with a higher exposure $(20 \%$ in case of the generic and $50 \%$ in case of the reference since it is formulation dependent). The failure to conclude equivalence does not seem to be due to a higher variability since the range of the $90 \% \mathrm{CI}$ of $\mathrm{C}_{\max }$ is similar. In summary, data generated using non-stereospecific assays may not be 
Table 1 Results of the bioequivalence analyses of test and reference formulations obtained by non-chiral bio-analytical method and chiral bioanalytical method ( $S$ - and $R$-ibuprofen)

\begin{tabular}{|c|c|c|c|c|}
\hline & Non-chiral & Sum of enantiomers & $S$-enantiomer & $R$-enantiomer \\
\hline $\mathrm{AUC}_{0-\mathrm{t}}$, point estimate $(\%)$ & 111.29 & 113.27 & 101.16 & 130.03 \\
\hline $\mathrm{AUC}_{0-\mathrm{t}}, 90 \% \mathrm{CI}(\%)$ & $107.23-115.49$ & $105.73-121.35$ & $96.67-105.86$ & $118.86-142.24^{\mathrm{a}}$ \\
\hline $\mathrm{AUC}_{0-\infty}$, point estimate $(\%)$ & 111.39 & 113.32 & 101.57 & 129.06 \\
\hline $\mathrm{AUC}_{0-\infty}, 90 \%$ CI (\%) & $107.29-115.65$ & $105.98-121.17$ & $97.18-106.16$ & $118.19-140.92^{\mathrm{a}}$ \\
\hline $\mathrm{C}_{\max }$, point estimate (\%) & 89.18 & 89.85 & 83.36 & 99.65 \\
\hline $\mathrm{C}_{\max }, 90 \% \mathrm{CI}(\%)$ & $82.77-96.09$ & $82.19-98.23$ & $76.05-91.36^{\mathrm{a}}$ & $87.84-113.05$ \\
\hline$t_{\max }$ of the test $(\mathrm{h})$ & 2.0 & 2.5 & 2.5 & 2.25 \\
\hline$t_{\max }$ of the reference $(h)$ & 0.75 & 0.875 & 1 & 0.75 \\
\hline
\end{tabular}

Test/reference ratios for AUC and $\mathrm{C}_{\max }$ based on log-transformed data are expressed as percentages

${ }^{\text {a }}$ Non-bioequivalent results

extrapolated to explain the exposure of the individual enantiomers [20].

Table 2 illustrates the concept of an enantiomer exposure ratio that is formulation dependent or absorption rate dependent. The $S / R$ AUC ratio showed a $34 \%$ difference (154 vs. $120 \%$ ) and the $S / R \mathrm{C}_{\max }$ ratio a $20 \%$ difference (121 vs. $101 \%$ ) between products. In our previous study [3] differences were smaller, $10 \%$ in AUC (123 vs. 114\%) and $6 \%$ in $\mathrm{C}_{\max }$ (112 vs. $106 \%$ ).

This study reminds that it is important to distinguish between non-linear kinetics (dose- or time-dependency) and non-linear absorption, as defined by the FDA [2], which may occur even in cases of linear kinetics, since ibuprofen shows non-linear kinetics due to saturation of protein binding only at doses greater than $600 \mathrm{mg}$. However, the inversion of $R$-ibuprofen to $S$-ibuprofen [21] is responsible of this non-linear absorption. This is consistent with the observations by Boni et al. [5], who showed that non-linear absorption may occur in a drug with linear pharmacokinetics in both the racemate and the individual enantiomers such as etodolac.

Table 2 AUC and $\mathrm{C}_{\max }$ data comparison regarding the enantiomer and drug formulation

\begin{tabular}{lll}
\hline & $\begin{array}{l}S / R \text { ratio } \\
\text { in test }\end{array}$ & $\begin{array}{l}S / R \text { ratio } \\
\text { in reference }\end{array}$ \\
\hline AUC $_{0-\text { t }}$ point estimate (\%) & 120.71 & 154.39 \\
AUC $_{0-\mathrm{t}}, 90 \%$ CI (\%) & $113.51-128.37$ & $146.13-163.12$ \\
$\mathrm{AUC}_{0-\infty}$, point estimate (\%) & 122.38 & 154.58 \\
$\mathrm{AUC}_{0-\infty}, 90 \%$ CI (\%) & $115.24-129.95$ & $146.28-163.35$ \\
$\mathrm{C}_{\text {max }}$, point estimate (\%) & 101.05 & 121.38 \\
$\mathrm{C}_{\text {max }}, 90 \%$ CI (\%) & $95.48-106.93$ & $114.95-128.18$ \\
$\mathrm{t}_{\max }$ of $S$-enantiomer (h) & 2.50 & 1.00 \\
$\mathrm{t}_{\max }$ of $R$-enantiomer (h) & 2.25 & 0.75 \\
\hline
\end{tabular}

It could be considered that ibuprofen enantiomers show similar pharmacokinetic characteristics based on selected literature data. For example, in our previous work the $S / R$ ratio was shown to be bioequivalent in that reference formulation (Neobrufen $600 \mathrm{mg}$ tablet) [3]. However, in the present study, the reference product (Dalsy $100 \mathrm{mg} / 5 \mathrm{~mL}$ oral suspension) showed a point estimate of $121 \%$ for $\mathrm{C}_{\max }$ and $154 \%$ for AUC, suggesting that the kinetics was not similar between the two enantiomers. Therefore, the similarity of pharmacokinetics between enantiomers should not be considered a compelling justification to use non-chiral bioanalytical methods, unless this similarity is thoroughly proven at a large range of different absorption rates.

Based on the results of this investigation it can be concluded that only two of the four FDA conditions have to be fulfilled to require a chiral bioanalytical method in bioequivalence studies. Measurement of individual enantiomers in bioequivalence studies should be recommended when (1) the enantiomers exhibit different pharmacodynamic characteristics, and (2) non-linear absorption is present, as expressed by a change in the enantiomer concentration ratio

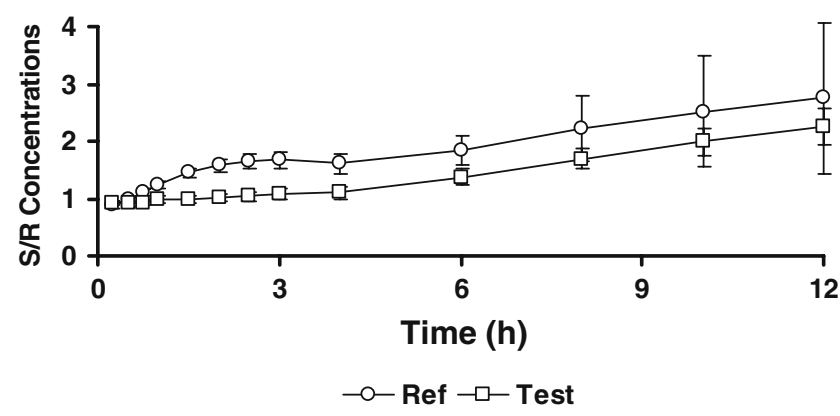

Fig. 4 Mean $S / R$ enantiomer plasma concentration ratio-time curve $(n=18)$ after oral administration of $600 \mathrm{mg}$ of ibuprofen in the reference and test formulations (calculated as mean of the individual ratios per time-point). Vertical bars show standard error of the mean 
with change in the input rate of the drug, for at least one of the enantiomers.

Acknowledgements This work was partially supported by a grant of the Complutense University and Santander to the research group 910939.

\section{Conflict of interest None}

\section{References}

1. Committee for Proprietary Medicinal Products (2001) Note for guidance on the investigation of bioavailability and bioequivalence. 26-7-2001. EMEA, London

2. US Department of Health and Human Services, Food and Drug Administration Center for Drug Evaluation and Research (CDER) (2003) Guidance for industry. Bioavailability and bioequivalence. Studies for orally adminsitered drug productsgeneral considerations. FDA CDER, Silver Spring, MD

3. Garcia-Arieta A, Abad-Santos F, Rodriguez-Martinez MA, VarasPolo Y, Novalbos J, Laparidis N, Gallego-Sandin S, Orfanidis K, Torrado J (2005) An eutomer/distomer ratio near unity does not justify non-enantiospecific assay methods in bioequivalence studies. Chirality 17(8):470-475

4. Committee for Proprietary Medicinal Products (CPMP) (1993) Note for guidance on the investigation of chiral active substances. EMEA, London

5. Boni JR, Korth-Bradley JM, Richards LS, Chiang ST, Hicks DR, Benet LZ (2000) Chiral bioequivalence: effect of absorption rate on racemic etodolac. Clin Pharmacokinet 39(6):459-469

6. Mehvar R, Jamali F (1997) Bioequivalence of chiral drugs. Stereospecific versus non-stereospecific methods. Clin Pharmacokinet 33(2):122-141

7. Geisslinger G, Stock KP, Bach GL, Loew D, Brune K (1989) Pharmacological differences between $\mathrm{R}(-)$-and $\mathrm{S}(+)$-ibuprofen. Agents Actions 27(3-4):455-457

8. Jamali F, Singh NN, Pasutto FM, Russell AS, Coutts RT (1988) Pharmacokinetics of ibuprofen enantiomers in humans following oral administration of tablets with different absorption rates. Pharm Res 5(1):40-43

9. Amidon GL, Lennernas H, Shah VP, Crison JR (1995) A theoretical basis for a biopharmaceutic drug classification: the correlation of in vitro drug product dissolution and in vivo bioavailability. Pharm Res 12(3):413-420

10. Lobenberg R, Amidon GL (2000) Modern bioavailability, bioequivalence and biopharmaceutics classification system. New scientific approaches to international regulatory standards. Eur J Pharm Biopharm 50(1):3-12

11. Lindenberg M, Kopp S, Dressman JB (2004) Classification of orally administered drugs on the World Health Organization model list of essential medicines according to the biopharmaceutics classification system. Eur J Pharm Biopharm 58(2): 265-278

12. Potthast H, Dressman JB, Junginger HE, Midha KK, Oeser H, Shah VP, Vogelpoel H, Barends DM (2005) Biowaiver monographs for immediate release solid oral dosage forms: ibuprofen. J Pharm Sci 94(10):2121-2131

13. Parojcic J, Corrigan OI (2008) Rationale for ibuprofen coadministration with antacids: potential interaction mechanisms affecting drug absorption. Eur J Pharm Biopharm 69(2):640-647

14. Fornasini G, Monti N, Brogin G, Gallina M, Eandi M, Persiani S, Bani M, Della PC, Zara G, Strolin BM (1997) Preliminary pharmacokinetic study of ibuprofen enantiomers after administration of a new oral formulation (ibuprofen arginine) to healthy male volunteers. Chirality 9(3):297-302

15. Farré M, Roset PN, Pascual JA, Abanades S, Manoyo E, Álvarez Y, Baena A (2005) Study on the bioavailability in extent and rate of ibuprofen tablets. Reumatol Clin 1(3):155-160

16. Chow SC, Liu JP (2000) Design and analysis of bioavailability and bioequivalence studies, 2nd edn. Marcel Dekker, New York

17. Chen ML, Lesko L, Williams RL (2001) Measures of exposure versus measures of rate and extent of absorption. Clin Pharmacokinet 40(8):565-572

18. Ring A, Tothfalusi L, Endrenyi L, Weiss M (2000) Sensitivity of empirical metrics of rate of absorption in bioequivalence studies. Pharm Res 17(5):583-588

19. Rostami-Hodjegan A, Jackson PR, Tucker GT (1994) Sensitivity of indirect metrics for assessing "rate" in bioequivalence studiesmoving the "goalposts" or changing the "game". J Pharm Sci 83 (11):1554-1557

20. Mehvar R (1994) Input rate-dependent stereoselective pharmacokinetics: effect of pulsatile oral input. Chirality 6(3):185-195

21. Brocks DR, Jamali F (1999) The pharmacokinetics of ibuprofen in humans and animals. In: Rainsford KD (ed) Ibuprofen. A critical bibliographical review. Taylor \& Francis, London, pp $90-142$ 